\title{
Avaliação dos Efeitos de Programas de Intervenção de Atividade Física em Indivíduos com Transtorno do Espectro do Autismo ${ }^{1}$ Assessment of the EFFects of Intervention Programs of Physical Activity IN INDIVIDUALS WITH AUTISM SPECTRUM DISORDER
}

\author{
Carla Cristina Vieira LOURENÇO² \\ Maria Dulce Leal ESTEVES ${ }^{3}$ \\ Rui Manuel Nunes CORREDEIRA ${ }^{4}$ \\ André Filipe Teixeira e SEABRA ${ }^{5}$
}

\begin{abstract}
RESUMO: a prática regular de atividade física influencia a saúde e o bem-estar, com papel importante na prevenção de várias doenças crônicas, como doenças cardiovasculares, acidente vascular cerebral, hipertensão, obesidade, diabetes, osteoporose, dentre outras. As vantagens da prática de exercício físico por pessoas com deficiências de desenvolvimento, especificamente de pessoas com transtorno do espetro do autismo (TEA), têm sido estudadas, mas dados as diferentes metodologias e abordagens presentes na literatura, há necessidade de fazer uma compilação das diferentes investigaçóes existentes que permitam concluir quais os efeitos de diferentes tipos de exercício em pessoas com TEA. O objetivo deste artigo é reunir os principais estudos que foram realizados nos últimos anos no âmbito da atividade física em indivíduos com (TEA) e retirar as conclusôes acerca dos mesmos. São apresentados 18 estudos, num total de 140 crianças e adultos com várias variantes desta síndrome e que participaram em atividades individualmente ou em grupo. Foram realizadas diversas atividades tais como jogos, natação, corrida, passeios terapêuticos, hidroginástica. As intervençôes pretenderam estudar a influência das atividades propostas no comportamento agressivo e estereotipado, funcionamento social, resistência, qualidade de vida e stress, aptidão física e resistência. Os programas de intervenção revelaram melhorias significativas, mostrando as potencialidades do exercício em pessoas com TEA.
\end{abstract}

PALAVRAS-CHAVE: Educação Especial. Atividade Física. Transtorno do Espectro do Autismo.

\begin{abstract}
Regular physical activity influences health and well-being, with an important role in the prevention of several chronic diseases, such as cardiovascular disease, stroke, hypertension, obesity, diabetes, osteoporosis. The advantages of physical exercise for people with developmental disabilities, specifically people with autism-spectrum disorder (ASD) have been studied, but given the different methodologies and approaches in the literature, there is the need to make a compilation of the different existing research that might allow us to conclude which are the effects of the different types of exercise in people with ASD. The purpose of this article is to bring together the main studies that have been conducted in the latest years in the context of physical activity in individuals with ASD and draw conclusions about them. Eighteen studies are presented, with a total of 140 children and adults with various variants of this syndrome, who participated in individual activities or in groups. Various activities, such as games, swimming, jogging, therapeutic walks, aerobics were performed. Interventions intended to study the influence of the activities proposed in aggressive and stereotyped behavior, social functioning, strength, quality of life and stress, physical fitness and endurance. Intervention programs revealed significant improvement, showing the benefits of exercise for people with ASD.
\end{abstract}

KEYWORDS: Special Education. Physical Activity. Autism Spectrum Disorder.

\footnotetext{
${ }^{1}$ http://dx.doi.org/10.1590/S1413-65382115000200011

${ }^{2}$ Departamento de Ciências do Desporto, Universidade da Beira Interior, Covilhã, Portugal. ccvl@ubi.pt

${ }^{3}$ Departamento de Ciências do Desporto, Universidade da Beira Interior, Centro de Investigação em Desporto, Saúde e Desenvolvimento Humano. Covilhã, Portugal. desteves@ubi.pt

${ }^{4}$ Departamento de Atividade Física Adaptada, Faculdade de Desporto da Universidade do Porto, Centro de Investigação em Atividade Física e Lazer, Porto, Portugal. rcorredeira@fade.up.pt

${ }^{5}$ Laboratório de Cineantropometria, Faculdade de Desporto da Universidade do Porto, Porto, Portugal. aseabra@fade.up.pt
} 


\section{INTRODUÇÁo}

As perturbaçóes do espectro do autismo consistem num distúrbio neurológico que se caracteriza por limitaçóes nas interaçóes sociais e de comunicação, de interesse restrito, e comportamentos estereotipados ou repetitivos (AMERICAN PSYCHIATRIC ASSOCIATION, 1994 apud DOWNEY; RAPPORT, 2012).

Apesar de serem de natureza semelhante, as perturbações do espectro do autismo inclui os seguintes diagnósticos distintos: autismo, síndrome de Asperger e Transtorno Invasivo do Desenvolvimento não-especificado (AMERICAN PSYCHIATRIC ASSOCIATION, 2000; TANGUAY et al., 1998 apud FOURNIER et al., 2010).

É do conhecimento geral que a atividade física, desde que praticada de forma adequada, influencia positivamente a saúde e o bem-estar, com papel importante na prevenção de várias doenças crônicas (doenças cardiovasculares, acidente vascular cerebral, hipertensão, obesidade, diabetes, osteoporose, etc.) (WARBURTO; NICOL; BREDIN, 2006; KLAVESTRAND; VINGARD, 2009).

A atividade física apresenta igualmente grandes benefícios para pessoas com incapacidades, podendo representar uma mais-valia na evolução de diferentes patologias. A utilização de atividades físicas e desportivas em crianças com autismo tem vindo a ser realizada e estudada. Nas duas últimas décadas o interesse pelos potenciais benefícios do exercício físico nas perturbações do espectro do autismo (PEA) tem aumentado, mas a pesquisa realizada nesta área é, ainda, escassa e baseada em pequenos grupos (SOWA; MEULENBROEK, 2012).

Assim, o uso do exercício físico como instrumento de desenvolvimento das crianças com autismo tem vindo a ser utilizado de forma crescente, sendo necessária investigação teórica que suporte esta utilização.

Os déficits motores são um núcleo potencial característico das perturbações do espectro do autismo e o tratamento desta patologia deve considerar intervençóes destinadas a melhorar esses déficits, incluindo o performance motor envolvidos com a coordenação motora (marcha, equilíbrio, funçóes do braço e planejamento do movimento) Fournier et al. (2010).

\section{Método}

Esta revisão consistiu numa análise sistemática de estudos resultantes de programas de intervenção motora.

Em cada estudo foram identificados o autor e ano do estudo, tipo de intervenção realizada, o número de intervenientes e as suas características, objetivos do estudo e os resultados obtidos.

\subsection{Procedimento de PeSQuisa}

Esta pesquisa foi realizada usando as bases de dados: Journal of Autism and Developmental Disorders, Research in Autism Spectrum Disorders, ScienceDirect, SpringuerLink, Google Scholar bases de dados das Universidades Portuguesas que lecionam cursos de desporto. 
As palavras-chave utilizadas na pesquisa foram perturbaçóes do espectro do autismo, atividade e exercício físico.

\subsection{CRitérios de inClusâo}

Para serem incluídos na nossa revisão, os estudos teriam que incluir pelo menos dois critérios de inclusão: 1) os participantes tinham que ser crianças ou adultos com o diagnóstico de Transtorno do Espectro do Autismo (AMERICAN PSYCHIATRIC ASSOCIATION, 2000); 2) os programas de intervenção utilizados tinham que envolver atividade física.

\subsection{Participantes}

Dos 18 estudos selecionados, todos os participantes dos grupos experimentais estavam diagnosticados com Perturbações do Espectro do Autismo de acordo com os critérios da DSM-IV (AMERICAN PSYCHIATRIC ASSOCIATION, 2000). Alguns dos estudos apresentados envolveram, também, grupo de controlo. No total participaram nas intervençóes 140 indivíduos com PEA, entre os dois e 39 anos, sendo majoritariamente do sexo masculino.

\subsection{Programas de interVenÇÃo}

Os programas de intervenção apresentados foram: natação $(\mathrm{n}=3)$, exercícios aquáticos $(n=3)$, corrida $(n=2)$, corrida e outras atividades $(n=5)$, caminhada na passadeira $(n=1)$, exercícios terapêuticas $(n=2)$, jogos $(n=1)$ e atividades de lazer $(n=1)$. As intervençóes pretenderam beneficiar o comportamento agressivo e estereotipado, funcionamento social, resistência, qualidade de vida e stress, aptidão física e resistência.

\section{Resultados}

Para demonstrar a importância do exercício físico em crianças com autismo fizemos uma pesquisa sobre intervençôes motoras realizadas com populaçóes autistas.

O Quadro 1 mostra-nos alguns estudos realizados no âmbito de programas de intervenção motora em indivíduos autistas.

São apresentados os autores dos estudos e ano de publicação, o tipo de intervenção realizada, o número de intervenientes, os objetivos do estudo e os resultados obtidos. 


\section{Tabela 1 - Resumo dos estudos realizados no âmbito da atividade física no autismo}

\begin{tabular}{|c|c|c|c|c|}
\hline Autor & $\begin{array}{l}\text { Tipo de inter- } \\
\text { vençáo }\end{array}$ & $\begin{array}{c}\text { Número de interve- } \\
\text { nientes }\end{array}$ & Objetivos & Resultados \\
\hline $\begin{array}{l}\text { Allison, Basile e } \\
\text { MacDonald et } \\
\text { al. (1991) }\end{array}$ & $\begin{array}{l}20 \text { minutos diários } \\
\text { de jogos durante } \\
\text { duas semanas numa } \\
\text { pista ao ar livre. }\end{array}$ & $\begin{array}{l}\text { um individuo do sexo } \\
\text { masculino, com } 24 \text { anos } \\
\text { e diagnosticado com } \\
\text { autismo }\end{array}$ & $\begin{array}{l}\text { Comparar os efeitos do } \\
\text { exercício antecedente e o } \\
\text { medicamento Lorazepam } \\
\text { no comportamento agressi- } \\
\text { vo de um homem autista. }\end{array}$ & $\begin{array}{l}\text { O comportamento agressi- } \\
\text { vo foi reduzido. }\end{array}$ \\
\hline $\begin{array}{l}\text { Best e Jones } \\
(1974)\end{array}$ & $\begin{array}{l}\text { Hidroginástica e } \\
\text { natação durante } \\
\text { 30m. }\end{array}$ & $\begin{array}{l}\text { quatro crianças com } \\
\text { autismo, entre os dois e } \\
\text { quatro anos }\end{array}$ & $\begin{array}{l}\text { Verificar a importância de } \\
\text { um programa de exercício } \\
\text { físico no tratamento do } \\
\text { autismo. }\end{array}$ & $\begin{array}{l}\text { Melhorias no comporta- } \\
\text { mento motor bruto. Houve } \\
\text { melhorias no Peabody Picture } \\
\text { Vocabulary Test, Test Merrill } \\
\text { Palmer Mental, e o Fels Esca- } \\
\text { la de Avaliação do Compor- } \\
\text { tamento. No Teste a Frostig } \\
\text { do Desenvolvimento, ou o } \\
\text { teste Gunzberg de Desenvol- } \\
\text { vimento Social não foram } \\
\text { detectadas melhorias. }\end{array}$ \\
\hline $\begin{array}{l}\text { Bass, Duchowny } \\
\text { e Llabre et al. } \\
(2009)\end{array}$ & $\begin{array}{l}\text { Uma sessão de pas- } \\
\text { seios terapêuticos } \\
\text { de uma hora por } \\
\text { semana, durante } \\
12 \text { semanas. }\end{array}$ & $\begin{array}{l}\text { Grupo experimental } \\
19 \text { participantes: duas } \\
\text { meninas e } 17 \text { meninos } \\
\text { entre os cinco e } 10 \text { anos. }\end{array}$ & $\begin{array}{l}\text { Avaliar os efeitos da } \\
\text { equitação no funcionamen- } \\
\text { to social da criança com } \\
\text { autismo. }\end{array}$ & $\begin{array}{l}\text { As crianças autistas expostas } \\
\text { à equitação terapêutica apre- } \\
\text { sentaram maior sensibilidade } \\
\text { sensorial, motivaçáo social e } \\
\text { menos desatenção, distração e } \\
\text { comportamentos sedentários. }\end{array}$ \\
\hline $\begin{array}{l}\text { Celiberti et al. } \\
\text { (1997) }\end{array}$ & $\begin{array}{l}\text { Durante três } \\
\text { semanas realização } \\
\text { de três ciclos de } \\
\text { seis minutos de } \\
\text { corrida e seis de } \\
\text { caminhada. }\end{array}$ & $\begin{array}{l}\text { um menino de cinco } \\
\text { anos com transtorno } \\
\text { autista }\end{array}$ & $\begin{array}{l}\text { Avaliar os efeitos de dois } \\
\text { níveis de exercício (andar } \\
\text { e jogging) na supressão } \\
\text { de autoestimulaçáo do com- } \\
\text { portamento de um menino } \\
\text { de cinco anos com autismo. }\end{array}$ & $\begin{array}{l}\text { A autoestimulação diminui } \\
\text { após corrida. Uma redução } \\
\text { no comportamento pertur- } \\
\text { bador foi observada em } 50 \% \\
\text { das sessōes. A caminhada não } \\
\text { teve efeitos. }\end{array}$ \\
\hline $\begin{array}{l}\text { Elliot et al. } \\
(1994)\end{array}$ & $\begin{array}{l}20 \text { minutos a } \\
\text { andar de bicicleta e } \\
\text { correr. }\end{array}$ & $\begin{array}{l}\text { seis adultos (três } \\
\text { homens e três mulheres) } \\
\text { com autismo e modera- } \\
\text { do ou profundo atraso } \\
\text { mental. }\end{array}$ & $\begin{array}{l}\text { Examinar os efeitos de } \\
\text { condiçóes de exercício } \\
\text { antecedente em comporta- } \\
\text { mentos mal adaptativos e } \\
\text { comportamentos estereoti- } \\
\text { pados em seis adultos com } \\
\text { autismo. }\end{array}$ & $\begin{array}{l}\text { Os comportamentos mal } \\
\text { adaptativos e estereotipados } \\
\text { foram significativamente } \\
\text { diminuídos após o exercício } \\
\text { vigoroso (corrida). }\end{array}$ \\
\hline $\begin{array}{l}\text { Fragala- } \\
\text {-Pinkham, } \\
\text { Haley e O'neil } \\
(2008)\end{array}$ & $\begin{array}{l}30-50 \text { m de exercí- } \\
\text { cios aquáticos, du- } \\
\text { rante } 14 \text { semanas }\end{array}$ & $\begin{array}{l}\text { seis meninos, três com } \\
\text { autismo e três com } \\
\text { transtorno global do } \\
\text { desenvolvimento }\end{array}$ & $\begin{array}{l}\text { Verificar a eficácia e } \\
\text { segurança de programa de } \\
\text { exercícios aeróbicos aquá- } \\
\text { ticos, sobre a resistência } \\
\text { cardiorrespiratória para as } \\
\text { crianças com deficiência. }\end{array}$ & $\begin{array}{l}\text { Significativa redução no tem- } \\
\text { po para completar uma meia } \\
\text { milha em corrida/caminhada. } \\
\text { A força muscular e flexibilida- } \\
\text { de não revelaram resultados } \\
\text { significativos. }\end{array}$ \\
\hline $\begin{array}{l}\text { Garcia-Villa- } \\
\text { misar e Dattilo } \\
(2010) \text {. }\end{array}$ & $\begin{array}{l}\text { um ano de ativida- } \\
\text { des de lazer }\end{array}$ & $\begin{array}{l}37 \text { participantes ( } 22 \text { do } \\
\text { sexo masculino e } 15 \text { do } \\
\text { feminino), com idades } \\
\text { entre os } 17-39 \text { anos com } \\
\text { autismo e Síndrome de } \\
\text { Asperger. }\end{array}$ & $\begin{array}{l}\text { Examinar os efeitos de } \\
\text { um programa de lazer na } \\
\text { qualidade de vida e stress } \\
\text { de indivíduos com com } \\
\text { transtornos do espectro do } \\
\text { autismo. }\end{array}$ & $\begin{array}{l}\text { O stress diminuiu significati- } \\
\text { vamente após o programa de } \\
\text { lazer. Também, a qualidade } \\
\text { de vida e a produtividade } \\
\text { melhoraram. } \\
\text { Integraçáo social e inde- } \\
\text { pendência não registraram } \\
\text { melhoria significativa. }\end{array}$ \\
\hline
\end{tabular}




\begin{tabular}{|c|c|c|c|c|}
\hline $\begin{array}{l}\text { Hameury } \\
(2010)\end{array}$ & $\begin{array}{l}\text { Uma hora de equo- } \\
\text { terapia assistida }\end{array}$ & $\begin{array}{l}\text { seis crianças autistas, en- } \\
\text { tre os cinco e sete anos }\end{array}$ & & $\begin{array}{l}\text { As crianças apresentaram me- } \\
\text { lhorias de } 52 \% \text { na pontuação } \\
\text { CEF total; taxas de melhoria } \\
\text { por aspetos avaliados foram: } \\
\text { atençấo }(22 \%) \text {, percepção } \\
32 \% \text {, tônus } 46 \% \text {, o ajuste do } \\
\text { motor } 86 \%, \% \text { imitação } 60 \text {, } \\
\text { emoçấo } 16 \% \text {, contacto } 37 \% \text {, } \\
\text { e de comunicaçáo } 24 \% \text {. }\end{array}$ \\
\hline $\begin{array}{l}\text { Kern, Koegel, } \\
\text { Dunlap (1984) }\end{array}$ & $\begin{array}{l}\text { Corrida durante } \\
15 \mathrm{~m} \text { e brincar } \\
\text { pegando a bola } \\
\text { durante } 15 \mathrm{~m} .\end{array}$ & $\begin{array}{l}\text { três crianças com idades } \\
\text { entre os sete e } 11 \text { anos, } \\
\text { com autismo. }\end{array}$ & $\begin{array}{l}\text { Verificar se o exercício } \\
\text { (exercícios leves ou vigoro- } \\
\text { sa) afeta os comportamen- } \\
\text { tos estereotipados. }\end{array}$ & $\begin{array}{l}\text { Exercícios vigorosos (corrida) } \\
\text { contribuíram mais para a re- } \\
\text { duçáo da estereotipia do que } \\
\text { os exercícios com bola. }\end{array}$ \\
\hline $\begin{array}{l}\text { Lochbam e } \\
\text { Crews (2003) }\end{array}$ & $\begin{array}{l}\text { três participantes } \\
\text { foram ensinados a } \\
\text { andar de bicicleta } \\
\text { estacionário e dois } \\
\text { foram ensinados } \\
\text { a levantar pesos } \\
\text { numa máquina (18 } \\
\text { sessōes de treino) } \\
\end{array}$ & $\begin{array}{l}\text { cinco indivíduos com } \\
\text { autismo e deficiência } \\
\text { mental leve entre os } 16 \text { e } \\
21 \text { anos. }\end{array}$ & $\begin{array}{l}\text { Realizar um programa de } \\
\text { treino seguindo diretrizes } \\
\text { padronizadas em indivíduos } \\
\text { com autismo para demons- } \\
\text { trar seu potencial. }\end{array}$ & $\begin{array}{l}\text { Todos os participantes me- } \\
\text { lhoraram entre } 33 \% \text { e } 50 \% \\
\text { o condicionamento aeróbio } \\
\text { O treino de força muscular } \\
\text { também revelou melhorias, } \\
\text { variando de } 12 \% \text { a } 47 \% \text {. }\end{array}$ \\
\hline $\begin{array}{l}\text { Nicholson et al. } \\
\text { (2011) }\end{array}$ & $\begin{array}{l}\text { Durante duas } \\
\text { semanas e três vezes } \\
\text { por semana, } 12 \\
\text { minutos de corrida. }\end{array}$ & $\begin{array}{l}\text { quatro crianças com } \\
\text { nove anos, } \\
\text { dois com autismo } \\
\text { altamente funcional e } \\
\text { dois com } \\
\text { Síndrome de Asperger. }\end{array}$ & $\begin{array}{l}\text { Verificar se a atividade física } \\
\text { antecedente melhorara } \\
\text { o empenho acadêmico e } \\
\text { verificar se os efeitos se } \\
\text { mantêm após a interrupção } \\
\text { da intervenção. }\end{array}$ & $\begin{array}{l}\text { Este estudo demonstra que } \\
\text { uma intervenção de atividade } \\
\text { física pode trazer um enorme } \\
\text { benefício para as crianças } \\
\text { diagnosticadas com autismo, } \\
\text { podendo resultar num maior } \\
\text { desempenho acadêmico. } \\
\text { O tempo de empenho aca- } \\
\text { dêmico aumentou durante o } \\
\text { exercício físico. }\end{array}$ \\
\hline $\operatorname{Pan}(2010)$ & $\begin{array}{l}10 \text { semanas, } 90 \\
\text { minutos de } \\
\text { exercícios aquáticos }\end{array}$ & $\begin{array}{l}16 \text { rapazes com idade } \\
\text { entre seis-nove anos com } \\
\text { (oito com autismo de } \\
\text { alto funcionamento e } \\
\text { oito com } \\
\text { Síndrome de Asperger) }\end{array}$ & $\begin{array}{l}\text { Determinar a eficácia de } \\
\text { um programa } 10 \text { semanas } \\
\text { de natação de exercícios } \\
\text { aquáticos nas habilidades } \\
\text { aquáticas e nos compor- } \\
\text { tamentos sociais de } 16 \\
\text { meninos com transtornos } \\
\text { do espectro do autismo. }\end{array}$ & $\begin{array}{l}\text { Foi registada uma significati- } \\
\text { va melhoria para um dos dois } \\
\text { grupos nas habilidades aquá- } \\
\text { ticas), que também mostrou } \\
\text { uma redução significativa no } \\
\text { comportamento anti-social. }\end{array}$ \\
\hline $\operatorname{Pan}(2011)$ & $\begin{array}{l}60 \text { minutos de } \\
\text { exercícios aquá- } \\
\text { ticos, e vezes por } \\
\text { semana, durante } \\
14 \text { semanas. }\end{array}$ & $\begin{array}{l}15 \text { crianças, com idades } \\
\text { entre sete e } 12 \text { anos } \\
\text { (autismo com alto fun- } \\
\text { cionamento e Síndrome } \\
\text { de Asperger) }\end{array}$ & $\begin{array}{l}\text { Este estudo avaliou a } \\
\text { eficácia de um programa } \\
\text { aquático de } 14 \text { semanas na } \\
\text { aptidão física e habilidades } \\
\text { aquáticas de crianças com } \\
\text { transtornos do espectro } \\
\text { autista e seus irmãos sem } \\
\text { deficiência. }\end{array}$ & $\begin{array}{l}\text { Todas as crianças mostraram } \\
\text { melhoras significativas na } \\
\text { força muscular, resistência } \\
\text { flexibilidade e aptidão cardio- } \\
\text { vascular e todas as fases das } \\
\text { habilidades aquáticas. }\end{array}$ \\
\hline $\begin{array}{l}\text { Pitetti et al. } \\
\text { (2010) }\end{array}$ & $\begin{array}{l}\text { nove meses de } \\
\text { caminhada na } \\
\text { passadeira. }\end{array}$ & $\begin{array}{l}\text { cinco adolescentes, três } \\
\text { do sexo masculino e } \\
\text { dois do sexo feminino, } \\
\text { com idades entre } 14 \text { e } \\
18 \text { anos e com autismo } \\
\text { severo. }\end{array}$ & $\begin{array}{l}\text { A eficácia de nove meses de } \\
\text { caminhada na passadeira na } \\
\text { capacidade de exercício e } \\
\text { índice de massa corporal em } \\
\text { adolescentes com autismo } \\
\text { severo. }\end{array}$ & $\begin{array}{l}\text { Após o programa, os } \\
\text { participantes mostraram } \\
\text { uma significativa redução do } \\
\text { índice de massa corporal. O } \\
\text { peso mostrou uma redução } \\
\text { năo-significativa. }\end{array}$ \\
\hline
\end{tabular}




\begin{tabular}{|c|c|c|c|c|}
\hline $\begin{array}{l}\text { Rogers; Hem- } \\
\text { meter e Wolery } \\
(2010)\end{array}$ & $\begin{array}{l}45-60 \text { minutos de } \\
\text { nataçáo. }\end{array}$ & $\begin{array}{l}\text { três meninos, com } \\
\text { quatro e cinco anos de } \\
\text { idade; }\end{array}$ & $\begin{array}{l}\text { Avaliar a eficácia do uso } \\
\text { de um procedimento de } \\
\text { atraso constante de tempo } \\
\text { para ensinar habilidades de } \\
\text { nataçáo fundamentais para } \\
\text { três crianças com autismo. }\end{array}$ & $\begin{array}{l}\text { Os meninos aprenderam } \\
\text { três habilidades de natação } \\
\text { novas. Os meninos } 1 \text { e } 2 \\
\text { necessitaram de oito sessōes } \\
\text { para dominar a habilidade } \\
\text { em primeiro lugar, mas } \\
\text { exigiu seis sessôes (o número } \\
\text { mínimo) para cada uma das } \\
\text { outras habilidades. O menino } \\
3 \text { dominou as três habilidades } \\
\text { em seis sessôes. }\end{array}$ \\
\hline $\begin{array}{l}\text { Rosenthal-Ma- } \\
\text { lek e Mitchell } \\
\text { (1997) }\end{array}$ & 20 minutos corrida & $\begin{array}{l}\text { cinco meninos, com } \\
\text { idades entre } 14 \text { e } 15 \\
\text { anos autismo }\end{array}$ & $\begin{array}{l}\text { Avaliar os efeitos dos exer- } \\
\text { cícios aeróbicos nos com- } \\
\text { portamentos autiestimu- } \\
\text { latórios e no desempenho } \\
\text { acadêmico de adolescentes } \\
\text { com autismo. }\end{array}$ & $\begin{array}{l}\text { Nos cinco meninos estudados } \\
\text { o comportamento autoesti- } \\
\text { mulatório reduziu o desem- } \\
\text { penho melhorou. Após a } \\
\text { corrida a condiçáo acadêmica } \\
\text { e o número de tarefas con- } \\
\text { cluídas tinham aumentado } \\
\text { significativamente. }\end{array}$ \\
\hline $\begin{array}{l}\text { Todd e Reid } \\
(2006)\end{array}$ & $\begin{array}{l}\text { seis meses de } \\
\text { atividade física } \\
\text { ao ar livre ( } 30 \\
\text { minutos. Raquetes } \\
\text { de neve e corrida / } \\
\text { caminhada) }\end{array}$ & $\begin{array}{l}\text { três adolescentes, com } \\
\text { idades entre } 15,16 \text { e } 20 \\
\text { anos autismo }\end{array}$ & $\begin{array}{l}\text { Avaliar os resultados de um } \\
\text { pacote de intervençóes na } \\
\text { participação de duas ativi- } \\
\text { dades físicas: raquetes de } \\
\text { neve e caminhada / corrida. }\end{array}$ & $\begin{array}{l}\text { Os três participantes aumen- } \\
\text { taram a distância que anda- } \\
\text { ram ou correram ao longo do } \\
\text { programa. }\end{array}$ \\
\hline $\begin{array}{l}\text { Yilmaz et al. } \\
(2004)\end{array}$ & $\begin{array}{l}10 \text { semanas de trei- } \\
\text { no de nataçáa, } 60 \\
\text { minutos, três vezes } \\
\text { por semana. }\end{array}$ & $\begin{array}{l}\text { uma criança com nove } \\
\text { anos com autismo }\end{array}$ & $\begin{array}{l}\text { Determinar os efeitos } \\
\text { de exercícios aquáticos e } \\
\text { natação no desempenho } \\
\text { motor e aptidão física, } \\
\text { assim como, observar o } \\
\text { comportamento de uma } \\
\text { criança autista, como ele se } \\
\text { familiariza com a piscina, e } \\
\text { observar o desenvolvimento } \\
\text { de habilidades iniciantes de } \\
\text { nataçáo em crianças } \\
\text { com autismo. }\end{array}$ & $\begin{array}{l}\text { Foram encontradas melhorias } \\
\text { no equilíbrio, agilidade, velo- } \\
\text { cidade, pontos de energia, } \\
\text { aperto de mão, força muscu- } \\
\text { lar das extremidades superior } \\
\text { e inferior, flexibilidade e } \\
\text { aptidão cardiovascular. O } \\
\text { comportamento autista/ } \\
\text { estereotipado também foi } \\
\text { alterado. }\end{array}$ \\
\hline
\end{tabular}

\section{DisCUSSÃo}

Perante os estudos apresentados é possível concluirmos que os diversos autores pretendiam verificar a influência de programas de intervenção motora em vários domínios: comportamentos sociais, comportamentos estereotipados, qualidade de vida, desempenho acadêmico e comportamento motor.

O tipo de intervenção praticada varia entre corrida, caminhada, natação, hidroginástica, bicicleta, atividades de lazer, levantamento de pesos e exercícios aquáticos.

Ao analisarmos os resultados obtidos nos diferentes estudos, são notórias as melhorias encontradas na população estudada após as várias semanas de intervenção.

Relativamente à amostra utilizada nos diversos estudos, é possível verificar que, de um modo geral, são grupos pequenos e nalguns casos com apenas um interveniente. Majoritariamente são crianças ou adolescentes diagnosticados com autismo. 
O exercício melhora a condição física e reduz os padróes de comportamento mal adaptativo (LANCIONI et al., 1998), estereotipados (LANCIONI; REILLY, 1998; ELLIOT et al., 1994; YILMAZ et al., 2004), o comportamento agressivo (ALLISON; BASILE; MACDONALD et al., 1991) e comportamento anti social (PAN, 2010).

O índice de massa corporal de dez adolescentes autistas reduziu após um programa de caminhada (PITETTI et al., 2007) e o desempenho acadêmico apresentou melhorias (NICHOLSON et al., 2011; ROSENTHAL-MALEK; MITCHELL, 1997).

Após doze semanas de passeios terapêuticos foi reduzida a desatenção e as habilidades sensoriais melhoraram (BASS; DUCHOWNY; LLABRE et al., 2009) e seis crianças autistas, também, apresentaram melhorias na atenção, percepção e comunicação (HAMEURY, 2010).

Relativamente à condição física, a prática atividade física melhora a flexibilidade e equilíbrio (YILMAZ et al., 2004), força muscular (LOCHBAUM et al., 2003; PAN, 2011), resistência, flexibilidade e aptidão cardiovascular (PAN, 2011), redução no tempo de percurso (FRAGALA-PINKHAM; HALEY; O’NEIL, 2008), aumento da distância que andaram ou correram (TODD; REID, 2006).

Perante a literatura publicada a atividade física nos indivíduos com perturbações do espectro do autismo é um aspeto bastante pertinente, revelando benefícios nos diferentes domínios, sendo significativa a influência do exercício em pessoas com autismo, quer ao nível da melhoria da sua condição física, quer na melhoria das capacidades cognitivas e sensoriais.

Os déficits motores são um núcleo potencial característico das perturbações do espectro do autismo e o tratamento desta patologia deve considerar intervençóes destinadas a melhorar esses déficits, incluindo o performance motor envolvidos com a coordenação motora (marcha, equilíbrio, funçóes do braço e planejamento do movimento) Fournier et al. (2010), pelo que o uso do exercício físico como instrumento de desenvolvimento das crianças com autismo tem vindo a ser utilizado de forma crescente, sendo necessária investigaçáo teórica que suporte esta utilização.

\section{REFERÊNCIAS}

ALLISON, D.V.; BASILE, V.C.; MacDONALD, R.B. Brief report: Comparative effects of antecedent exercise and lorazepam on the aggressive behaviour of an autistic man. Journal of Autism and Developmental Disorders, v.21, n.3, p.379-384, 1991.

AMERICAN PSYCHIATRIC ASSOCIATION. Diagnostic and Statistical Manual of Mental Disorders, fourth edition, text revision (DSM-IV-TR). Washington: American Psychiatric Association, 2000.

BASS, M. M.; DUCHOWNY, C. A.; LLABRE, M. M. The effect of therapeutic horseback riding on social functioning in children with autism. Journal of Autism and Developmental Disorders, v.39, n.9, p.1261-1267, 2009.

BEST, J.; JONES, J. Movement therapy in the treatment of autistic children. Australian Occupational Therapy Journal, Austrália, v.21, n.2, p.72-86, 1974. 
CELIBERTI, D. A. et al. The differential and temporal effects of antecedent exercise on the selfstimulatory behavior of a child with autism. Research in Developmental Disabilities, Bethesda, v.18, n.2, p.139-150, 1997.

DOWNEY, R.; RAPPORT, M. J. K. Motor Activity in Children With Autism. Pediatric Physical Therapy, v.24, n.1, p.2-20, 2012.

ELLIOTT, R. O. et al. Vigorous, aerobic exercise versus general motor training activities: Effects on maladaptive and stereotypic behaviors of adults with both autism and mental retardation. Journal of Autism and Developmental Disorders, v.24, n.5, p.565-576, 1994.

FOURNIER, K. A. et al. coordination in autism spectrum disorders: A synthesis and meta-analysis. Journal of Autism and Developmental Disorders, v.40, n.10, p.1227-1240, 2010.

FRAGALA-PINKHAM, M.; HALEY, S. M.; O’NEIL, M. E. Group aquatic aerobic exercise for children with disabilities. Developmental Medicine and Child Neurology, v.50, n.11, p.822-827, 2008.

GARCIA-VILLAMISAR, D. A.; DATTILO, J. Effects of a leisure programme on quality of life and stress of individuals with ASD. Journal of Intellectual Disability Research, v.54, p.611-619, 2010.

HAMEURY, L. Équithérapie et autism. Annales Médico-Psychologiques, v.168, p.655-659, 2010.

KERN, L.; KOEGEL, R. L.; DUNLAP, G. The influence of vigorous versus mild exercise on autistic stereotyped behaviors. Journal of autism and developmental disorders, v.14, n.1, p.57-67, 1984.

KLAVESTRAND, J.; VINGÅRD E. The relationship between physical activity and health-related quality of life: a systematic review of current evidence. Scand J Med Sci Sports, v.19, n.3, p. 300-312, 2009.

LANCIONI, G. E.; REILLY, M. F. O. A Review of Research on Physical Exercise with People with Severe and Profound Developmental Disabilities. Science, v.19, n.6, p.477-492, 1998.

LOCHBAUM, M.; CREWS, D. Viability of Cardiorespiratory and Muscular Strength Programs for the Adolescent with Autism. Complementary Health Practice Review, v.8, n.3, p. 225-233, 2003.

NICHOLSON, H. et al. The effects of antecedent physical activity on the academic engagement of children with autism spectrum disorder. Psychology in the Schools, v.48, n.2, p.198-213, 2011.

PAN, C. Y. Effects of water exercise swimming program on aquatic skills and social behaviors in children with autism spectrum disorders. Autism, v.14, n.1, p.9-28, 2010.

PAN, C. Y. The efficacy of an aquatic program on physical fitness and aquatic skills in children with and without autism spectrum disorders. Research in Autism Spectrum Disorders, v.5, n.1, p.657-665, 2011.

PITETTI, K. H. et al. The efficacy of a 9-month treadmill walking program on the exercise capacity and weight reduction for adolescents with severe autism. Journal of Autism and Developmental Disorders, v.37, n.6, p.997-1006, 2007.

ROSENTHAL-MALEK, A.; MITCHELL, S. Brief Report : The Effects of Exercise on the SelfStimulatory Behaviors and Positive Responding of Adolescents with Autism. Journal of autism and developmental disorders, v. 27, n.2, p.193-202, 1997.

ROGERS, L.; HEMMETER, M.; WOLERY, M. Using a Constant Time Delay Procedure to Teach Foundational Swimming Skills to Children With Autism. Topics in Early Childhood Special Education, v.30, p.1-10, 2010. 
SOWA, M.; MEULENBROEK, R. Research in Autism Spectrum Disorders Effects of physical exercise on Autism Spectrum Disorders: A meta-analysis. Research in Autism Spectrum Disorders, v.6, n.1, p.46-57, 2012.

TODD, T.; REID, G. Increasing Physical Activity in Individuals With Autism. Focus on Autism and Other Developmental Disabilities, v.21, n.3, p.167-176, 2006.

YILMAZ, I. et al. Effects of swimming training on physical fitness and water. Pediatrics Internacional, v.46, p.624-626, 2004.

Recebido em: 25/02/2015

Reformulado em: 22/05/2015

Aprovado em: 10/06/2015 
LOURENÇO, C.C.V. et al. 